\title{
DÉFICIT DE VOLUME DE LÍQUIDOS: PERFIL DE CARACTERÍSTICAS DEFINIDORAS NO PACIENTE PORTADOR DE QUEIMADURA
}

\author{
Lídia Aparecida Rossi* \\ Maria Célia B. Dalri** \\ Ana Emília Pace Ferraz*** \\ Emilia Campos de Carvalho**** \\ Miyeko Hayashida*****
}

ROSSI, L.A.; DALRI, M.C.B.; FERRAZ, A.E.P.; CARVALHO, E.C.de; HAYASHIDA, M. Déficit de volume de líquidos: perfil de características definidoras no paciente portador de queimadura. Rev.latino-am.enfermagem, Ribeirão Preto, v. 6, n. 3, p. 85-94, julho 1998.

O objetivo deste estudo foi descrever o perfil das características definidoras do paciente com diagnóstico de enfermagem "Déficit de volume de líquidos" relacionado a perda ativa de líquidos, secundária à queimadura. Os dados foram coletados através de um instrumento, contendo 29 características definidoras possiveis para esse diagnóstico, aplicado a sete enfermeiros, que trabalhavam há pelo menos cinco anos em serviços especializados em tratamento de queimaduras. Os enfermeiros avaliaram as características definidoras, quanto ao grau que cada uma é indicativa desse diagnóstico, atribuindo valores numa escala de zero a um. Os resultados confirmaram todas as caracteristicas definidoras apresentadas pela North American Nursing Diagnosis Association (NANDA), exceto uma (temperatura corporal elevada), e apontaram 10 novas características.

Unitermos: diagnóstico de enfermagem, queimaduras, déficit de volume de líquidos

\section{INTRODUÇÃO}

As alterações decorrentes do Déficit do Volume de Líquidos provocam mudanças no funcionamento de todos os sistemas orgânicos. O enfermeiro deve estar apto para detectar essas alterações precocemente com o intuito de prevenir o agravamento da situação que pode acontecer de forma súbita ou gradual. Concordamos com GERSHAN et al. ${ }^{7}$ quando afirmam que a identificação dessas alterações pode ser difícil porque seus indicadores são contraditórios na literatura. Desta forma, em um primeiro momento, nos empenhamos em realizar um estudo, com o objetivo de determinar quais os possíveis sinais e sintomas que refletem a presença de Déficit de Volume de Líquidos, em particular entre pessoas portadoras de queimaduras.

O diagnóstico de enfermagem Déficit de Volume de Líquidos é definido por GERSHAN et al. ${ }^{7}$, como "um estado em que o indivíduo pode apresentar um desequilíbrio nos fluídos corporais por entrada deficiente de líquidos ou perdas excessivas" e pela North American Nursing
Diagnosis Association (NANDA) ${ }^{14}$, como o "estado em que o indivíduo experimenta uma desidratação vascular, celular ou intracelular, resultante de falhas nos mecanismos reguladores e/ou perda ativa de volume de líquidos".

A água corporal total está distribuída tanto no interior da célula (fluído intracelular) como fora desta (fluído extracelular), sendo que o fluido extracelular está dividido em três compartimentos: intersticial, intravascular e transcelular. A troca dinâmica dos fluídos ocorre continuadamente entre os compartimentos intracelular, extracelular (plasma) e intersticial. Destes três compartimentos, somente o extracelular é diretamente influenciado pela ingesta ou eliminação de fluídos do corpo. Alterações nos compartimentos dos fluidos intracelular e intersticial ocorrem em resposta à mudanças no volume ou concentração do plasma (CHENEVEY) ${ }^{2}$.

O volume dos fluídos corporais é mantido por uma interação precisa entre a entrada e saída através dos rins, pela perda através da pele e pulmões, em função da pressão hidrostática no interior do compartimento vascular

\footnotetext{
* Enfermeira, Assistente junto ao Departamento de Enfermagem Geral e Especializada da Escola de Enfermagem de Ribeirão Preto - Universidade de São Paulo (DEGE, EERP-USP)

** Enfermeira, Diretora Técnica do Serviço de Centro Cirúrgico e Material da Unidade de Emergência do Hospital das Clínicas da Faculdade de Medicina de Ribeirão Preto, USP *** Enfermeira, Professora Doutora junto ao DEGE, EERP-USP **** Enfermeira, Professora Titular junto ao DEGE, EERP-USP ***** Enfermeira, Chefe da Secção de Apoio Laboratorial da EERP-USP
} 
e da osmolaridade dentro de cada compartimento. O rim desempenha papel fundamental na regulação da concentração da maior parte dos constituintes do líquido extracelular (GUYTON) ${ }^{9}$.

A regulação da concentração do íon sódio é controlada principalmente, pela regulação da quantidade de água do corpo. Quando a concentração de sódio aumenta, ativa dois mecanismos, cujos efeitos têm como finalidade aumentar a quantidade de água no corpo: secreção de hormônio antidiurético $(\mathrm{ADH})$ pela glândula hipófise posterior e estimulação da sede. O teor aumentado de água no corpo dilui o sódio no líquido extracelular e faz com que sua concentração decresça até o normal (GUYTON) ${ }^{9}$.

Por outro lado, quando a concentração do íon potássio eleva-se, dois outros mecanismos de controle por "feedbak" negativo entram em ação. Primeiro é o efeito direto do potássio em excesso sobre as células epiteliais dos túbulos renais, produzindo transporte aumentado de potássio dos capilares peritubulares para o lúmen tubular, de onde os íons potássio são perdidos na urina. O segundo mecanismo de "feedback" é a estimulação do córtex supra-renal para secretar quantidades muito elevadas de aldosterona, em resposta a concentração aumentada do íon potássio. Este mecanismo estimula as células epiteliais tubulares a transportar potássio para o lúmen tubular, de onde será perdido pela urina (GUYTON) ${ }^{9}$.

A falha de um dos mecanismos supra citados pode levar a um aumento do débito urinário e conseqüentemente à desidratação. McFARLAND \& McFARLENE $^{12}$ citam algumas situações patológicas que podem resultar em falhas nos mecanismos controladores do volume dos líquidos corporais. Assim, o estado de coma, pode neutralizar a sensação de sede. O "diabetes insipidus", que pode ser provocado por traumatismos ou cirurgias, situações inflamatórias, tumores, resulta numa deficiência do $\mathrm{ADH}$, podendo também provocar desidratação pelo aumento do débito urinário. Outras situações patológicas como a doença de Addison causa redução de aldesterona, e diante da insuficiência de aldosterona o sódio não é retido, mas sim excretado levando água consigo em quantidades aumentadas. $\mathrm{Na}$ cetoacidose diabética, quando há descontrole do "diabetes mellitus", a diminuição de insulina resulta em hiperglicemia, glicosúria e cetoacidose, conseqüentemente, há aumento da osmolaridade no espaço intravascular, provocando a entrada de água do espaço intersticial e intracelular para o espaço intravascular, que é excretada pelos rins e, eventualmente, pode provocar desidratação (McFARLAND \& McFARLENE) $)^{12}$.

Quando há falha nos mecanismos reguladores a manifestação clínica do diagnóstico de déficit de volume de líquidos será caracterizada por: aumento do volume urinário, com urina diluída, inesperada alteração de peso, densidade urinária em torno de 1000 a 1005 . O volume urinário pode chegar a 15 ou 16 litros em 24 horas. $\mathrm{O}$ paciente pode ainda apresentar hipotensão e diminuição do enchimento venoso, taquicardia, pele seca, diminuição do turgor e aumento da temperatura. O hematócrito e a hemoglobina poderão estar elevados (McFARLAND \& McFARLENE) ${ }^{12}$. De forma geral, esse processo ocorre em pacientes independente de terem sofrido trauma térmico.

Nos pacientes vítimas de queimadura o déficit do volume de líquidos ocorre, predominantemente, através da perda de fluídos para fora do corpo e para o espaço intersticial em função do traumatismo. O déficit de volume de líquidos relacionado à perda ativa de fluídos pode ser provocado também por vômitos, diarréias e outros tipos de traumatismos, além da queimadura. $\mathrm{O}$ paciente com diarréia perde sódio e potássio e pode desenvolver acidose. O paciente com vômitos perde sódio, ácido clorídrico, potássio e magnésio e pode desenvolver alcalose. (McFARLAND \& McFARLENE) $^{12}$.

Os tipos de fluídos perdidos podem ser plasma, sangue ou líquido extracelular. A perda de sangue pode ocorrer principalmente em situações de traumatismos abertos ou fechados. O plasma geralmente é perdido em queimaduras, em traumas por esmagamentos e processos inflamatórios. As desidratações ocorrem em função das perdas dos líquidos extracelulares. Ao diminuir o volume sangüíneo ele se torna insuficiente para suprir os tecidos e o equilíbrio circulatório é rompido com a diminuição do débito cardíaco e pressão arterial. Através de um mecanismo compensatório o organismo lança mão da atividade adrenérgica instalando-se a vasoconstricção periférica e uma isquemia no leito capilar (GALLUCCI) ${ }^{6}$.

O diagnóstico de déficit de volume de líquidos poderá estar caracterizado por diminuição do volume urinário com aumento de sua concentração e uma densidade maior que 1001, perda súbita de peso, elevação do hematócrito e alterações do sódio sérico. Outras características incluem: sede, aumento da freqüência de pulso, diminuição do turgor da pele, hipotensão e pele seca (McFARLAND \& McFARLENE) ${ }^{12}$.

É importante considerar que dependendo do fator relacionado ao diagnóstico, as características definidoras poderão divergir em alguns aspectos. Assim, na coleta e na análise dos dados deverão ser consideradas essas diferenças, muitas vezes, bastante sutis.

Em um estudo realizado por ROSSI et al. ${ }^{15}$, que teve como objetivo caracterizar a aplicação dos diagnósticos de enfermagem em uma Unidade de Queimados, foi constatado que $64,7 \%$ dos pacientes estudados apresentaram o diagnóstico de déficit de volume de líquidos. DALRI ${ }^{3}$ identificou esse diagnóstico em $82,4 \%$ dos pacientes portadores de queimadura. 
Nesses estudos, os diagnósticos foram estabelecidos nas primeiras 72 horas após o trauma.

O paciente portador de queimaduras sofre uma grande perda de fluidos pela passagem de plasma do compartimento intravascular para o espaço intersticial. Essa perda é proporcional a extensão e profundidade da lesão $(\mathrm{KING})^{11}$. Essa situação ocorre em função do aumento da permeabilidade capilar, diminuição da pressão colóido-osmótica vascular, e uma alteração na pressão hidrostática capilar.

Com a dilatação, os capilares se tornam porosos ocorrendo, assim, saída de eletrólitos, água, plasma para fora do vaso, em torno dos tecidos que funcionam como uma esponja, formando o edema. A perda normal diária varia de $700 \mathrm{ml}$ a $1000 \mathrm{ml}$ por dia. O paciente grande queimado perde aproximadamente $350 \mathrm{ml}$ por hora, sendo que a maior perda ocorre dentro das primeiras 12 a 18 horas transcorridas após o acidente (DYER \& ROBERTS $)^{4}$. TOCANTINS \& GOMES $^{17}$ ressaltam que o aumento da permeabilidade capilar chega a permitir a saída de partículas com peso molecular de 150.000 , quando em condições normais nem a albumina de peso molecular 60000 atravessa os poros. Segundo os autores, como conseqüência da grande perda de volemia e de mecanismos neurohumorais indefinidos, quando o consumo de oxigênio figura abaixo dos níveis normais, pode ocorrer hipotensão com tendência ao choque, ocorrendo ainda um aumento da freqüência cardíaca e aumento da resistência periférica.

Ao longo da perda de fluidos e, conseqüentemente, da hipovolemia o paciente queimado tem um desequilíbrio eletrolítico. A aldosterona é secretada em função da hipovolemia e promove a liberação de sódio e a reabsorção de água nos túbulos renais. Essa retenção de sódio provoca rapidamente a retenção de água no sistema vascular. Adicionalmente há uma falha na bomba de sódio, que normalmente funciona bombeando sódio para fora da célula em troca de potássio para dentro, resultando em liberação de potássio para o espaço intravascular proveniente das células teciduais e da hemólise das células destruídas pelo trauma. A secreção urinária do potássio é prejudicada pela diminuição da perfusão e função renal. Depois de 24 horas a pressão hidrostática no espaço intersticial começa a dificultar a saída de fluido dos capilares, a membrana capilar é restaurada e ocorre diminuição da perda de fluidos (MONAHAN et al.) ${ }^{13}$.

Considerando que os indicadores clínicos podem apresentar diferenças dependendo da condição do paciente e do fator relacionado ao diagnóstico, esse estudo tem como objetivo descrever o perfil das características definidoras do paciente portador de queimaduras com déficit de volume de líquidos.

\section{METODOLOGIA}

Para realização deste estudo foi elaborado um instrumento composto por 29 características definidoras para o diagnóstico déficit de volume de líquidos, estabelecidas a partir das características definidoras apresentadas pela NANDA ${ }^{14} \mathrm{e}$ acrescidas de outras identificadas na literatura (MONAHAN et al. ${ }^{13}$; GERSHAN et al. ${ }^{7}$; GALLUCCI) ${ }^{6}$, focalizando o paciente portador de queimaduras. Na taxionomia I da NANDA ${ }^{14}$, são consideradas como características definidoras maiores para esse diagnóstico: mudança no débito urinário, mudança na concentração da urina, enchimento venoso diminuído, hemoconcentração, alteração no sódio sérico e súbita perda ou ganho de peso. Dessas características definidoras mudança no débito urinário e mudança na concentração da urina foram alteradas, respectivamente, para: diminuição do débito urinário e aumento da concentração da urina, visando torná-las mais específicas para o paciente portador de queimaduras. A NANDA ${ }^{14}$ apresenta como características definidoras menores: hipotensão, sede, freqüência de pulso aumentada, diminuição do turgor da pele, diminuição do volume e da pressão do pulso, temperatura corporal elevada, pele seca, membranas e mucosas secas, fraqueza e mudança no estado mental.

Em um primeiro momento foi realizada a validação do instrumento quanto a aparência e conteúdo por cinco enfermeiros. Esses enfermeiros propuseram pequenas modificações quanto ao conteúdo que foram incorporadas ao instrumento. As orientações para o preenchimento, bem como o objetivo do presente estudo estavam contidas no instrumento (Anexo 1).

Posteriormente, foi verificada a relevância atribuída por enfermeiros a cada evidência clínica possível de ser observada em pacientes queimados. Para o desenvolvimento desta etapa empregamos os passos sugeridos por $\mathrm{FEHRING}^{5}$ para realização de validação de conteúdo de um diagnóstico. Essa proposta compreende apenas uma parte de um processo mais amplo de validação*. Os passos desenvolvidos nesse estudo estão descritos a seguir:

1. Foi solicitado a sete enfermeiros, que trabalhavam há pelo menos 5 anos em serviços especializados em

* Sobre validação de Diagnóstico de Enfermagem sugerimos a leitura de:

INVITATIONAL CONFERENCE ON RESEACH METHODS FOR VALIDATING NURSING DAGNOSES, Palm Springs, California, North American Nursing Diagnosis Association e Case Western Reserve University, Frances Payne Bolton School of Nursing, 1989 
tratamento de pacientes portadores de queimaduras, que analisassem as características definidoras contidas no instrumento.

Para cada evidência clínica, os enfermeiros deveriam escolher uma entre cinco alternativas que indicavam o quanto cada evidência caracterizava o diagnóstico déficit de volume de líquidos relacionado ao trauma térmico, a saber, muitíssimo, muito, de algum modo, pouco e nada característico.

2. Para cada opção de resposta foram atribuídos valores da seguinte forma: muitíssimo $=1$; muito $=0,75$; de algum modo $=0,5 ;$ pouco $=0,25 ;$ nada $=0$. A partir dos escores obtidos para cada característica definidora foi calculada a média aritmética para cada uma das evidências.

3. De acordo com a proposta de FEHRING ${ }^{5}$, as características definidoras com média menor ou igual a 0,50 foram desconsideradas. Entretanto, o autor recomenda, que nesse caso, outros estudos sejam realizados para confirmação ou não, desses resultados. 4. As evidências clínicas com peso maior ou igual a 0,80 foram consideradas características definidoras maiores, e as com peso entre 0,50 a 0,799 foram consideradas como características menores. Da mesma forma que no passo anterior, estudos complementares são necessários para a confirmação ou não desses resultados.

5. O escor total foi obtido através do cálculo da média dos resultados obtidos no item 2, para características definidoras maiores e menores respectivamente.

\section{RESULTADOS E DISCUSSÃO}

$\mathrm{Na}$ Tabela 1, a seguir estão apresentados os resultados referentes as características definidoras consideradas maiores pela NANDA ${ }^{14}$ para o diagnóstico déficit de volume de líquidos.

Tabela 1 - Distribuição das características definidoras apresentadas pela NANDA $^{14}$ como maiores, conforme as médias de escores atribuídos pelos enfermeiros, com respectivos desvios padrão, para o diagnóstico de déficit de volume de líquidos

\begin{tabular}{l|c|c}
\hline \multicolumn{1}{c|}{$\begin{array}{c}\text { Características definidoras } \\
\text { maiores da NANDA }\end{array}$} & $\begin{array}{c}\text { Escores } \\
\text { médios }\end{array}$ & $\begin{array}{c}\text { Desvio } \\
\text { padrão }\end{array}$ \\
\hline Diminuição do débito urinário & 1,0 & 0 \\
\hline $\begin{array}{l}\text { Aumento na concentração da } \\
\text { urina }\end{array}$ & 0,96 & 0,087 \\
\hline Enchimento venoso diminuído & 0,96 & 0,087 \\
\hline Alteração no sódio sérico & 0,83 & 0,117 \\
\hline Hemoconcentração & 0,78 & 0,208 \\
\hline Súbito ganho ou perda de peso & 0,61 & 0,323 \\
\hline
\end{tabular}

Os resultados deste estudo confirmam a proposta da taxionomia da NANDA ${ }^{14}$, mantendo a diminuição do volume urinário, o aumento da concentração da urina, o enchimento venoso diminuído e a alteração no sódio sérico como características definidoras maiores (Tabela 1).

Embora a hemoconcentração seja considerada como uma característica definidora maior pela NANDA ${ }^{14}$, neste estudo, os resultados indicam que esta evidência seria uma característica menor (Tabela 1). Como resultado da grande perda de líquidos para o espaço extravascular, o paciente portador de queimaduras apresenta um aumento na viscosidade do sangue que pode estar evidenciado no aumento do hematócrito. Pode-se observar que entre os enfermeiros não houve uma concordância expressiva com relação a esse resultado. É possível que essa situação resulte de uma falta de atenção por parte de alguns enfermeiros com relação aos resultados de exames laboratoriais ou que o aumento da concentração do sangue não seja suficiente para expressar resultados considerados anormais no hematócrito. Outros estudos necessitam ser realizados para esclarecer essa questão. Por essa razão, consideramos importante manter essa evidência como uma característica definidora maior conforme proposto pela NANDA ${ }^{14}$.

Como resultado da presença de grandes volumes no espaço extravascular o paciente queimado, muitas vezes, pode não apresentar perda de peso nas primeiras horas após o trauma, o que justifica os resultados que indicam que a súbita perda ou ganho de peso deveria ser considerada como uma característica definidora menor (Tabela 1).

Na Tabela 2, a seguir, estão apresentadas as características definidoras que não são mencionadas pela Taxionomia da NANDA ${ }^{14} \mathrm{e}$ foram apontadas no presente estudo como maiores.

Tabela 2 - Distribuição das características definidoras não apresentadas pela NANDA ${ }^{14}$, classificadas neste estudo como maiores, conforme as médias de escores atribuídos pelos enfermeiros, com respectivos desvios padrão, para o diagnóstico de déficit de volume de líquido

\begin{tabular}{l|c|c}
\hline \multicolumn{1}{c|}{$\begin{array}{c}\text { Características } \\
\text { definidoras }\end{array}$} & $\begin{array}{c}\text { Escores } \\
\text { médios }\end{array}$ & $\begin{array}{c}\text { Desvio } \\
\text { padrão }\end{array}$ \\
\hline Edema & 0,93 & 0,112 \\
\hline Ansiedade & 0,89 & 0,23 \\
\hline $\begin{array}{l}\text { Diminuição da pres são venosa } \\
\text { central }\end{array}$ & 0,82 & 0,174 \\
\hline $\begin{array}{l}\text { Aumento da freqüência } \\
\text { respiratória }\end{array}$ & 0,82 & 0,112 \\
\hline
\end{tabular}


Após o trauma térmico há um aumento da permeabilidade capilar ocasionando a saída de elementos não celulares, ions inorgânicos, água plasmática e proteínas de alto peso molecular do espaço intravascular e extraceleular para o compartimento extravascular. A passagem desses elementos resulta na diminuição da pressão capilar-osmótica plasmática, provocando uma perda maior de líquidos dos vasos para o espaço intersticial nas áreas não queimadas, levando a hipovolemia, edema e diminuição da pressão venosa central (ARTZ et al.) ${ }^{1}$.

Com base nos resultados obtidos, apresentados na Tabela 2, entendemos que o edema deve ser considerado como uma característica definidora maior nos casos de pacientes que sofreram trauma térmico, como um sinal que indica a perda de líquido. Entretanto, entendemos que esse sinal deverá estar associado a presença de outros, pois isoladamente pode não ser suficiente para a indicação do diagnóstico de déficit de volume de líquidos.

A diminuição da pressão venosa central também foi considerada uma evidência clínica importante pelos enfermeiros. Desse modo, entendemos que essa evidência deve ser considerada como uma característica definidora maior na Taxionomia da NANDA ${ }^{14}$, entretanto, não pode ser uma característica definidora crítica. Isto porque, como ressalta GOMES et al. ${ }^{8}$, nas primeiras vinte e quatro horas após o trauma térmico a pressão venosa central é igual a zero, mas esse dado não é um bom parâmetro, pois indica apenas a situação do compartimento direito do coração, não refletindo a volemia.

Os resultados deste estudo indicam que a ansiedade deveria ser considerada como evidência maior desse diagnóstico. $\mathrm{O}$ paciente portador de queimaduras apresenta-se ansioso. Entretanto, considerando que essa situação é uma manifestação do paciente frente a experiência do trauma, que é singular para cada pessoa, pois envolve fatores associados ao próprio trauma (idade, extensão da superfície corporal queimada, ambiente, causas do acidente e a personalidade do paciente) e fatores associados ao estresse fisiológico, outros estudos necessitam ser realizados para confirmar ou não esse resultado.

$\mathrm{Na}$ Tabela 3, a seguir, estão apresentadas as características definidoras consideradas pela Taxionomia da NANDA ${ }^{14}$ como menores.
Tabela 3 - Distribuição das características definidoras apresentadas pela NANDA $^{14}$ como menores, conforme as médias de escores atribuídos pelos enfermeiros, com respectivos desvios padrão, para o diagnóstico de déficit de volume de líquido

\begin{tabular}{l|c|c}
\hline $\begin{array}{l}\text { Características definidoras } \\
\text { menores da NANDA }\end{array}$ & $\begin{array}{c}\text { Escores } \\
\text { médios }\end{array}$ & $\begin{array}{c}\text { Desvio } \\
\text { padrão }\end{array}$ \\
\hline Sede & 0,89 & 0,123 \\
\hline Membranas e mucosas secas & 0,86 & 0,123 \\
\hline Freqüência de pulso aumentada & 0,83 & 0,117 \\
\hline Diminuição do volume do pulso & 0,82 & 0,112 \\
\hline Pele seca & 0,75 & 0,327 \\
\hline Hipotensão & 0,71 & 0,159 \\
\hline Dirninuição da pressão do pulso & 0,71 & 0,159 \\
\hline Mudança do turgor da pele & 0,64 & 0,349 \\
\hline Mudança no estado mental & 0,64 & 0,123 \\
\hline Fraqueza & 0,61 & 0,123 \\
\hline Temperatura corporal elevada & 0,50 & 0,204 \\
\hline
\end{tabular}

A verbalização de sede é uma característica definidora, apresentada pela NANDA ${ }^{14}$ como menor, mas os resultados obtidos indicam que essa deveria ser incluída como uma evidência maior desse diagnóstico. Essa proposta está respaldada na literatura, pois a verbalização de sede tem sido considerada, por muitos autores, como o primeiro sinal de desidratação celular.

A pele seca e a diminuição do turgor da pele são considerados pela NANDA como características definidoras menores desse diagnóstico e, da mesma forma, pelos enfermeiros neste estudo, entretanto, com uma variação maior entre as respostas. É provável que essa situação esteja relacionada a dificuldade de avaliação desses sinais no paciente portador de extensas áreas queimadas (Tabela 3 )

A diminuição do volume do pulso é um sinal que tem sido apontado na literatura como indicativo da diminuição da volemia, e foi considerado como características definidora maior nesse estudo.

A temperatura corporal elevada é uma evidência considerada menor pela taxionomia da NANDA ${ }^{14}$. Neste estudo, o aumento da temperatura corporal obteve um escore médio de 0,50 (Tabela 3 ). O paciente queimado apresenta-se hipotérmico, pois apresenta dificuldade em manter a temperatura corporal nos níveis de normalidade em função da perda da microcirculação nas áreas lesadas (SMELTZER \& BARE) $^{16}$. Considerando essa situação e os resultados obtidos nesse estudo, a temperatura corporal elevada não foi incluída como uma característica 
que compõe o perfil do diagnóstico déficit de volume de líquidos relacionado a perda ativa de líquidos secundária ao trauma térmico.

$\mathrm{Na}$ Tabela 4, a seguir são apresentadas as características definidoras não apresentadas pela NANDA $^{14}$ como uma evidência desse diagnóstico, mas consideradas, de acordo com os resultados deste estudo, como evidências menores deste diagnóstico.

Tabela 4 - Distribuição das características definidoras não apresentadas pela NANDA ${ }^{14}$, classificadas neste estudo como menores, conforme as médias de escores atribuídos pelos enfermeiros, com respectivos desvio padrão, para o diagnóstico de déficit de volume de líquido

\begin{tabular}{l|c|c}
\hline $\begin{array}{l}\text { Caracteristicas definidoras } \\
\text { menores propostas neste } \\
\text { estudo }\end{array}$ & $\begin{array}{c}\text { Escores } \\
\text { médios }\end{array}$ & $\begin{array}{c}\text { Desvio } \\
\text { padräo }\end{array}$ \\
\hline Pele fria & 0,78 & 0,154 \\
\hline Agitação & 0,75 & 0,113 \\
\hline Aumento da Hemoglobina & 0,68 & 0,290 \\
\hline Hipotensão postural & 0,67 & 0,276 \\
\hline Letargia & 0,64 & 0,123 \\
\hline $\begin{array}{l}\text { Aumento da profundidade } \\
\text { respiratória }\end{array}$ & 0,61 & 0,182 \\
\hline Palidez & 0,57 & 0,323 \\
\hline
\end{tabular}

A hipotensão postural pode ser uma manifestação clínica inicial quando ocorre a perda ativa de fluidos. Quando instalado e persistente, o déficit de volume de líquidos pode levar o paciente ao choque. $\mathrm{O}$ paciente poderá apresentar diminuição do retorno venoso, do débito cardíaco, da perfusão e da função dos órgãos vitais, principalmente do cérebro, rins e coração (MONAHAN et al. ${ }^{13}$ ). Como manifestações clínicas dessas situações o paciente poderá apresentar palidez, letargia, pele fria, hipotensão, aumento da profundidade respiratória e fraqueza. Os resultados laboratoriais também poderão indicar aumento da hemoglobina em função da diminuição do volume intravascular no paciente portador de queimaduras (IGNAVICIUS et al. ${ }^{10}$ ).

$\mathrm{Na}$ Figura 1, a seguir, apresentamos a proposta de perfil das características definidoras maiores, para o paciente com déficit de volume de líquidos. Nas propostas apresentadas consideramos o escor total obtido nesse estudo que foi de 0,88 .
Figura 1 - Proposta de características definidoras maiores para o diagnóstico déficit de volume de líquidos relacionado a perda ativa de fluidos, secundário ao trauma térmico

\begin{tabular}{l}
\hline Características definidoras maiores \\
\hline Diminuição do débito urinário* \\
\hline Aumento da concentração da urina* \\
\hline Enchimento venoso diminuido* \\
\hline Hemoconcentração* \\
\hline Alteração no sódio sérico* \\
\hline Membranas e mucosas secas** \\
\hline Sede** \\
\hline Frequência de pulso aumentada** \\
\hline Diminuição do volume do pulso** \\
\hline Diminuição da pressão venosa central \\
\hline Edema \\
\hline Aumento da frequência respiratória \\
\hline * Características definidoras apresentadas pela Taxionomia I da \\
NANDA como maiores \\
**Características definidoras apresentadas pela Taxionomia I da \\
NANDA como menores \\
As características não sinalizadas não são citadas na Taxionomia I da \\
NANDA
\end{tabular}

A Figura 2 apresenta as propostas realizadas nesse estudo para características definidoras menores para o diagnóstico déficit de volume de líquidos relacionado a perda ativa de líquidos, secundária ao trauma térmico. $\mathrm{O}$ escor total obtido foi de 0,68 para as características menores. Consideramos que esse índice pode ser útil para a realização de outros estudos complementares.

Figura 2 - Proposta de características definidoras menores para o diagnóstico déficit de volume de líquidos relacionado a perda ativa de fluidos, secundário ao trauma térmico

\begin{tabular}{l}
\hline Características definidoras menores \\
\hline Súbito garho ou perda de peso* \\
\hline Pele seca** \\
\hline Hipotensão** \\
\hline Diminuição da pressão do pulso ${ }^{* *}$ \\
\hline Diminuição do turgor da pele** \\
\hline Mudança no estado mental ${ }^{* *}$ \\
\hline Fraqueza** \\
\hline Pele fria \\
\hline Aumento da Hemoglobina \\
\hline Hịotensão postural \\
\hline Letargia \\
\hline Palidex \\
\hline Aumento da profundidade respiratória \\
* Características definidoras apresentadas pela Taxionomia I da NANDA \\
como maiores \\
**aracterísticas definidoras apresentadas pela Taxionomia I da NANDA \\
como menores \\
As características não sinalizadas não são citadas na Taxionomia I da \\
NANDA
\end{tabular}




\section{CONSIDERAÇÕES FINAIS}

O perfil das características definidoras para o paciente com diagnóstico de Déficit de volume de líquidos relacionado a perda ativa de líquidos, secundária ao trauma térmico, estudado com base nos passos do modelo proposto por FEHRING ${ }^{5}$, tem como características definidoras maiores: diminuição do débito urinário, aumento da concentração da urina, enchimento venoso diminuído, edema, hemoconcentração, alteração no sódio sérico, membranas e mucosas secas, sede, freqüência de pulso aumentada, diminuição da pressão venosa central, diminuição do volume do pulso, ansiedade e aumento da freqüência respiratória. Destas características definidoras, não são mencionadas pela $\operatorname{NANDA}^{14}$ as que se seguem: edema, aumento da freqüência respiratória e diminuição da pressão venosa central, porém entendemos que deveriam ser consideradas como evidências maiores deste diagnóstico. Foram também consideradas como outras evidências maiores deste diagnóstico: membranas e mucosas secas, sede, freqüência de pulso aumentada e diminuição do volume do pulso. Destacamos que essas características são apontadas pela NANDA ${ }^{14}$ como características definidoras menores. Assim, das seis características definidoras apresentadas pela NANDA ${ }^{14}$ como maiores, uma foi considerada como uma evidência menor e as restantes foram confirmadas como maiores. Foram também apresentadas quatro novas características definidoras maiores.

Entre as características definidoras apontadas na Taxionomia da NANDA ${ }^{14}$ não estão incluídas as seguintes: pele fria, palidez, aumento da hemoglobina, hipotensão postural, letargia e aumento da profundidade respiratória. Essas evidências clínicas foram consideradas, neste estudo, como características definidoras menores. Os resultados indicam que o súbito ganho ou a perda de peso, apontado pela taxionomia como uma evidência maior, deve ser incluído como uma característica definidora menor. Das 10 características definidoras apontadas pela NANDA ${ }^{14}$ como menores, cinco foram confirmadas, uma foi considerada como não indicativa desse diagnóstico (temperatura corporal elevada) e quatro foram consideradas como maiores. Foram também apresentadas seis novas características definidoras menores.

Esses resultados compõem o conjunto de características definidoras do diagnóstico déficit de volume de líquidos relacionado a perda ativa de líquidos, secundária ao trauma térmico; consideramos importante que outros estudos de validação clínica sejam realizados com o paciente portador de queimaduras para confirmar ou modificar as alterações propostas neste estudo.

\section{FLUIDVOLUME DEFICIT: PROFILE OF DEFINING CHARATERISTICS IN BURNT PATIENTS}

The present study aimed at describing the profile of defining characteristics in patients with the nursing diagnosis "Fluid volume deficit" related to active loss of fluid secondary to burns. Data were collected by means of a tool, containing 29 possible defining characteristics of this diagnosis. Seven nurses, that worked at the Burnt Unit for at least five years ago, provided opinions about the degree to which each defining characteristic is indicative of this diagnosis. Nurses rated each defining characteristic of diagnosis being tested on a scale of zero to one. The results confirmed all, except one (increased body temperature) defining characteristics presented by NANDA for this diagnosis and indicated 10 new defining characteristics.

KEY WORDS: nursing diagnosis, burns, fluid volume deficit

\section{DÉFICIT DE VOLUMEN DE LÍQUIDOS: PERFIL DE CARACTERÍSTICAS QUE LA DEFINEN EN PACIENTES PORTADORES DE QUEMADURAS}

El objetivo de este estudio fue describir el perfil de las características que la definen en paciente con diagnóstico de enfermería "Déficit de volumen de líquidos" relacionado a la pérdida activa de líquidos, secundaria a quemadura. La recolección de datos fue hecha a través de un instrumento que conttene 29 características posibiles que definen ese diagnóstico. El instrumento fue desarrollado con siete enfermeros que trabajaban en una Unidad de Quemados, los quales poseían una experiencia mínima de cinco años. Los enfermeros evaluaron las características en cuanto al grado que cada una de estas características eran indicativas de ese diagnóstico, atribuyendo valores en una escala de zero a uno. Los resultados confirmaron todas las características presentadas por NANDA para ese diagnóstico, excepto una (temperatura corporal elevada) y indicaron 10 nuevas características.

TÉRMINOS CLAVES: diagnóstico de enfermería, quemaduras, déficit de volumen de líquidos 


\section{ANEXO 1 \\ UNIVERSIDADE DE SÃO PAULO \\ ESCOLA DE ENFERMAGEM DE RIBEIRÃO PRETO \\ DEPARTAMENTO DE ENFERMAGEM GERAL E ESPECIALIZADA}

\section{DÉFICIT DE VOLUME DE LÍQUIDOS EM PACIENTES PORTADORES DE QUEIMADURAS: considerações teóricas e validação do diagnóstico de enfermagem}

\section{INSTRUÇÕES:}

Este instrumento faz parte de um estudo destinado a validar as características definidoras (sinais e sintomas) do diagnóstico de enfermagem Déficit de Volume de Líquidos relacionado a perda ativa de líquidos, secundária ao trauma térmico, cuja definição se encontra na página seguinte.

O objetivo deste instrumento é averiguar o quanto estas características que têm estado presentes quando você presta assistência de enfermagem para pessoas adultas com esse problema.

Nas páginas seguintes você encontrará itens representando tais características.

Solicitamos que leia atentamente a cada uma delas e assinale, com base em sua experiência, sua concordância ou discordância quanto à inclusão de cada característica definidora.

Os itens se encontram no extremo da esquerda e as cinco possibilidades de resposta à direita. Você deve indicar apenas uma das possibilidades. Assim, as cinco possibilidades de respostas são:

\begin{tabular}{|c|c|c|c|c|}
\hline $\begin{array}{c}\text { Este item é } \\
\text { muitíssimo } \\
\text { característico }\end{array}$ & $\begin{array}{c}\text { Este item é } \\
\text { muito } \\
\text { característico }\end{array}$ & $\begin{array}{c}\text { Este item é de } \\
\text { algum modo } \\
\text { característico }\end{array}$ & $\begin{array}{c}\text { Este item é } \\
\text { pouco } \\
\text { característico }\end{array}$ & $\begin{array}{c}\text { Este item é } \\
\text { nada } \\
\text { característico }\end{array}$ \\
\hline
\end{tabular}

É muito importante que você responda todas as proposições.

Por favor assinale há quanto tempo você presta assistência a pacientes portadores de queimaduras: anos.

Muito obrigada pela colaboração.

Déficit de Volume de Líquidos: estado no qual o indivíduo apresenta volume vascular diminuído, desidratação celular ou intersticial.

\begin{tabular}{|c|c|c|c|c|c|}
\hline Item & $\begin{array}{c}\text { muitís } \\
\text { simo }\end{array}$ & muito & $\begin{array}{c}\text { de } \\
\text { algum } \\
\text { modo }\end{array}$ & pouco & nada \\
\hline Diminuição do débito urinário (menor que 30 ml/hora) & & & & & \\
\hline Aumento da concentração da urina & & & & & \\
\hline Enchimento venoso diminuído & & & & & \\
\hline Pressão venosa central (menor que $4 \mathrm{~cm}$ de $\mathrm{H}_{2} \mathrm{O}$ ) & & & & & \\
\hline $\begin{array}{l}\text { Hemoconcentração (hematócrito - homem = } 40 \text { a } 54 \% \text {; mulher } \\
=37 \mathrm{a} 47 \% \text { ) }\end{array}$ & & & & & \\
\hline $\begin{array}{l}\text { Aumento da hemoglobina }(\text { homem }=15 \mathrm{~g} / 100 \mathrm{ml} ; \text { mulher }=12 \\
\text { a } 14 \mathrm{~g} / 100 \mathrm{ml} \text { ) }\end{array}$ & & & & & \\
\hline
\end{tabular}




\begin{tabular}{|c|c|c|c|c|c|}
\hline Item & $\begin{array}{c}\text { muitís } \\
\text { simo }\end{array}$ & muito & $\begin{array}{c}\text { de } \\
\text { algum } \\
\text { modo }\end{array}$ & pouco & nada \\
\hline \multicolumn{6}{|l|}{ Edema } \\
\hline \multicolumn{6}{|c|}{ Ateração no sódio sérico (136 a 145 mEq/l) } \\
\hline \multicolumn{6}{|c|}{ Hipotensão ( pressão sistólica menor que 90 mmHg) } \\
\hline \multicolumn{6}{|c|}{ Hipotensão postural } \\
\hline \multicolumn{6}{|c|}{ Freqüência de pulso aumentada (acima de 100 bat./min.) } \\
\hline \multicolumn{6}{|l|}{ Arritmia } \\
\hline \multicolumn{6}{|c|}{ Diminuição da pressão do pulso } \\
\hline \multicolumn{6}{|c|}{ Diminuição do volume do pulso } \\
\hline \multicolumn{6}{|c|}{ Aumento da freqüência respiratória (maior que $20 / \mathrm{mov} . / \mathrm{min}$.) } \\
\hline \multicolumn{6}{|c|}{ Aumento da profundidade respiratória } \\
\hline \multicolumn{6}{|c|}{ Perda de peso (maior ou igual a $5 \%$ do peso total) } \\
\hline \multicolumn{6}{|c|}{ Temperatura corporal elevada (maior ou igual a $37,8^{\circ} \mathrm{C}$ ) } \\
\hline \multicolumn{6}{|l|}{ Sede } \\
\hline \multicolumn{6}{|l|}{ Pele fria } \\
\hline \multicolumn{6}{|c|}{ Diminuição do turgor da pele (pastoso e semipastoso) } \\
\hline \multicolumn{6}{|l|}{ Pele seca } \\
\hline \multicolumn{6}{|c|}{ Mucosas secas } \\
\hline \multicolumn{6}{|l|}{ Palidez } \\
\hline \multicolumn{6}{|l|}{ Fraqueza } \\
\hline \multicolumn{6}{|l|}{ Letargia } \\
\hline \multicolumn{6}{|c|}{ Confusão mental } \\
\hline \multicolumn{6}{|l|}{ Agitação } \\
\hline Ansiedade & & & & & \\
\hline
\end{tabular}

Outros - especificar:

\begin{tabular}{|l|l|l|l|l|l|}
\hline & & & & & \\
\hline & & & & & \\
\hline & & & & & \\
\hline & & & & & \\
\hline
\end{tabular}


Você gostaria de acrescentar algum aspecto do tema que não foi abordado?
( ) Não
() $\mathrm{Sim}$

Coloque aqui os seus comentários e/ou sugestões:

Ribeirão Preto - setembro, 1996.

\section{REFERÊNCIAS BIBLIOGRÁFICAS}

01. ARTZ, C.P.; MONCRIEF, J.A.; PRUITT, B.A. Queimaduras. Rio de Janeiro: Interamericana, 1980.

02. CHENEVEY, B. Overview of fluids and eletrolytes. Nurs. Clin. North Am., v. 2, n. 4, p. 749-59, 1987.

03. DALRI, M. C. Perfil diagnóstico de pacientes queimados segundo o modelo conceitual de Horta e a Taxonomia I Revisada da NANDA. Ribeirão Preto, 1993. Dissertação (Mestrado) Escola de Enfermagem de Ribeirão Preto, Universidade de São Paulo.

04. DYER, C. ; ROBERTS, D. Thermal trauma. Nurs. Clin. North Am., v, 25, n. 1, p. 85-117, 1990.

05. FEHRING, R. Methods to validate nursing diagnosis. Heart Lung, v. 17, p. 625-9, 1987.

06. GALLUCCI, C. Choque. 2. ed. São Paulo: Editora de Publicações Médicas, 1985.

07. GERSHAN, J. A. et al. Fluid volume deficit: Validating the indicators. Heart \& Lung, v.19, n. 2, p. 152-6, 1990.

08. GOMES, D. R.; SERRA, M. C. ; PELLON, M. A. Queimaduras. Rio de Janeiro: Livraria e Editora Revinter, 1995.

09. GUYTON, A. C. Fisiologia humana. 6. ed. Rio de Janeiro: Guanabara Koogan, 1988.
10. IGNATAVICIUS, D. D. ; WORKIMAN, M. L.; MSHLER, M. A. Medical surgical nursing: a nursing process approach. 2 ed. Philadelphia: W. B. Saunders, 1995.

11. KING, M. W. Nursing consideration of the burned patient during the emergent period. Heart Lung, v.11,p. 353-61, 1982.

12. McFARLAND, G. K. ; McFARLENE, E A Nursing diagnosis \& intervention: planning for patient care. St Louis: CV Mosby, 1989.

13. MONAHAN, F.; DRAKE, T.; NEIGHBORS, M. Nursing care of adults. Philadelphia: Saunders, 1994.

14. NANDA. Nursing Diagnosis: definitions \& classification. Philadelphia: North American Nursing Diagnosis Association, 1996.

15. ROSSI, L. A. et al. Aplicação dos diagnósticos de enfermagem em Unidade de Queimados. In: SEMINÁRIO NACIONAL DE PESQUISA EM ENFERMAGEM, 8, Ribeirão Preto, 10 a 13 de julho de 1995. Programa. Ribeirão Preto: Associação Brasileira de Enfermagem, 1995. p.12.

16. SMELTZER, S. C. ; BARE, B. Brunner/Suddarth: tratado de enfermagem médico-cirúrgica. 7. ed. Rio de Janeiro: Guanbara Koogan, 1994. v. 3.

17. TOCANTINS, R. GOMES, D. R. Grande queimado. Prát. Hosp., v.1, n.4, p. 6-10, 1986. 